\title{
Sumo Robot Research
}

\author{
Miao zhiquan \\ Department of Electronics and Information, North China Institute of Science and Technology, Yanjiao, Beijing-east, \\ (miaozhiq@ncist.edu.cn)
}

\begin{abstract}
In recent years, with of robot technology progress and robot science activities, robot technology obtained fast development. Now robot technology has been widely applied to measure, monitor, industrial control, diagnosis, scientific experiments each domain. The system USES the Atmega128 single-chip Atmel company as a core controller, was designed using a infrared to tube detection boundary, looking for each other, controller to tube receiving infrared data, and according to the data control motor state thus robot reached automatic control purposes. Against robot by single-chip microcomputer smallest system, detection circuit and motor drive circuit structure and realizes looking for each other, and will launch the right-field other functions.
\end{abstract}

Keywords-Infrared to tube, detection circuit, motor drive

\section{相扑机器人的研制探讨}

\author{
苗志全 \\ 华北科技学院电子信息工程学院, 东燕郊, 北京, 中国
}

摘 要 近年来随着机器人技术的进步和机器人科普活动的开展, 机器人技术得到了快速的发展。如今机器人技术已经被广泛地 应用到测量、监测、工业控制、诊断、科学试验等各个领域中。本系统采用 Atmel 公司的 Atmega128 单片机作为核心控制器, 设计了一 种利用红外对管检测边界、寻找对方, 控制器接收红外对管数据, 并根据数据控制电机的状态, 从而机器人达到了自动控制的目的。 相扑机器人由单片机最小系统、检测电路和电机驱动电路等构成, 实现了寻找对方、并将对方推出界外的等功能。

关键词 红外对管、检测电路、电机驱动

\section{1. 引言}

近年来随着机器人技术的进步和机器人科普活动的开 展, 机器人技术得到了快速的发展。机器人技术已经被广 泛地应用到测量、监测、工业控制、诊断、科学试验等各 个领域中。例如, 当监测一些对人体有害的环境时, 工作 人员现场监测就可能对人的生命构成危害, 这时我们可以 利用机器人监测、并将数据带回, 然后工作人员查看数据, 这样可以保证工作人员安全使得工作顺利进行。

本项目所研制为相扑机器人, 即机器人能够模仿人 寻找对方, 并将对方推出界外。相扑机器人属于表演型 机器人的范畴, 考虑到大学生对机器人的了解不多, 相 扑机器人可以作为教学使用。

\section{2. 作品结构框图及工作原理}

2.1 作品结构框图

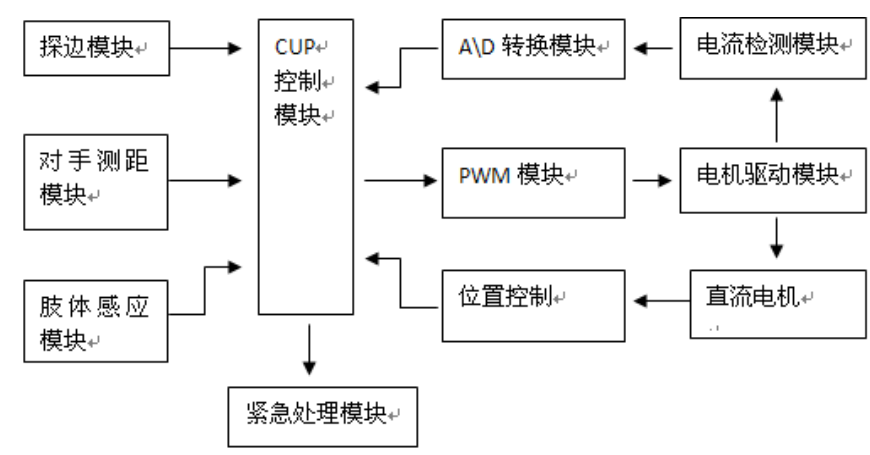

图 2-1 相扑机器人

公益性科研院所基本科研业务费专项资金资助项目 


\section{2 工作原理}

单片机最小系统由电源模块、独立按键电路和显示电 路组成; 检测电路由红外对管组成; 电机驱动采用 LN298 模块。其中检测电路包括边界检测红外对管和寻找对方红 外对管两部分。

机器人在对抗过程中, 首先, 由边界检测红外对管检 测机器人是否出界: 当机器人前面将要出界时, 立即改变 机器人当前运行状态, 使机器人后退; 反之, 使机器人前 进。然后, 在机器人没有出界的情况下, 机器人旋转寻找 对方。在寻找到对方后攻击地方, 直到一方将令一方退出 界为止。

\section{3. 硬件设计}

本系统由单片机最小系统、检测电路和电机驱动电路 组成[1]。

\section{1 单片机最小系统}

单片机最小系统主要包扩电源模块、复位和时钟模 块、按键模块和显示模块[2]。

\section{1 .1 电源模块}

电源模块是一个由 $12 \mathrm{~V}-5 \mathrm{~V}$ 双电源电路。考虑到机器 人的电机对速度和力矩的要求, 在电机全速前进时电机两 端电压外加 $12 \mathrm{~V}$ 直流电压。而单片机最小系统及其他外围 为器件所需要的都是 $5 \mathrm{~V}$ 直流电压, 所以, 我们选用 7805 三端稳压芯片将 $12 \mathrm{~V}$ 直流电压变换成 $5 \mathrm{~V}$ 。12V 直流电源用 笔记本的锂电池, 笔记本电池电流最大可以达到 $2 \mathrm{~A}$, 可以 满足电机的需要。

\section{1 .2 复位和时钟模块}

复位采用常用的上电自动复位和按钮复位两种方式; 时钟采用外接晶体和电容组成的并联谐振回路。

\subsection{3 按键模块}

常用的按键模块有矩阵键盘和独立键盘。考虑到本系 统使用独立键盘可以满足需要, 所以本系统中按键模块采 用独立键盘。

\section{1 .4 显示模块}

在嵌入式开发过程中常用的显示模块有 LED、LCD 和点阵等, 本系统采用 LED 数码管, 因为 LED 数码管廉 价, 而且能够满足本系统需要。

\section{2 检测电路}

检测电路包括检测边界和检测对方两个方面。当检测 边界的红外对管检测到黑线时为高电平, 否则为低电平,
这样只有两种状态, 检测距离短, 但能够满足检测边界要 求, 程序处理也比较简单。

而检测对方需要红外对管检测距离长, 常用的有超声 波检测、红外编码检测、红外对管检测等等[3]。但前两种 方案程序比较复杂, 所以, 本系统采用红外对管检测。单 片机普通 IO 口接红外对管只能检测十几厘米, 当双方距离 较远时不能寻找到对方。但是, 红外对管输出的是模拟信 号, 如果将红外对管输出的模拟信号进行模数转换, 这样 只要红外对管输出的模拟信号有很微小的变化单片机就可 以检测到, 检测距离可以到几十厘米, 甚至一米, 可以满 足系统要求, 而且 $\mathrm{AD}$ 程序比较简单。所以, 我们采用此 种方案, 电路图如图 3-2-1 所示。这也是我们系统的亮点之 一。
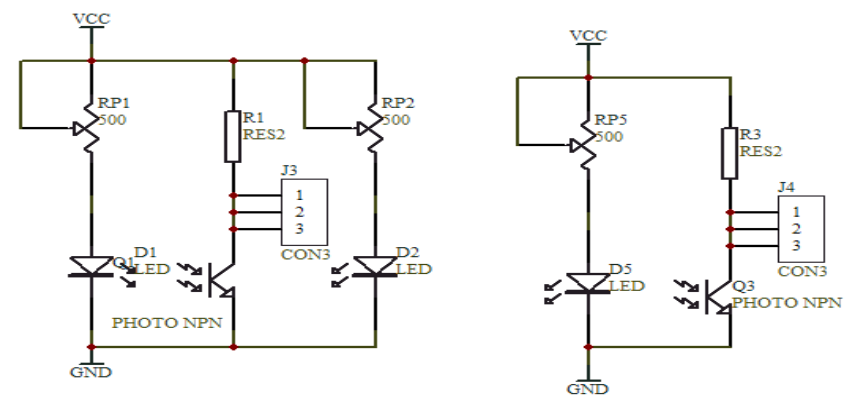

图 3-1 检测电路

\section{3 电机驱动电路}

采用电机专用驱动芯片, 如 LN298、MC33886 等。专 用驱动芯片电路简单、克服了分立元件电路的不对称性问 题; LN298 最大承受电流达到 2A, 而且性能稳定, 可以满 足系统要求。电机驱动电路如图 3-2 所示。

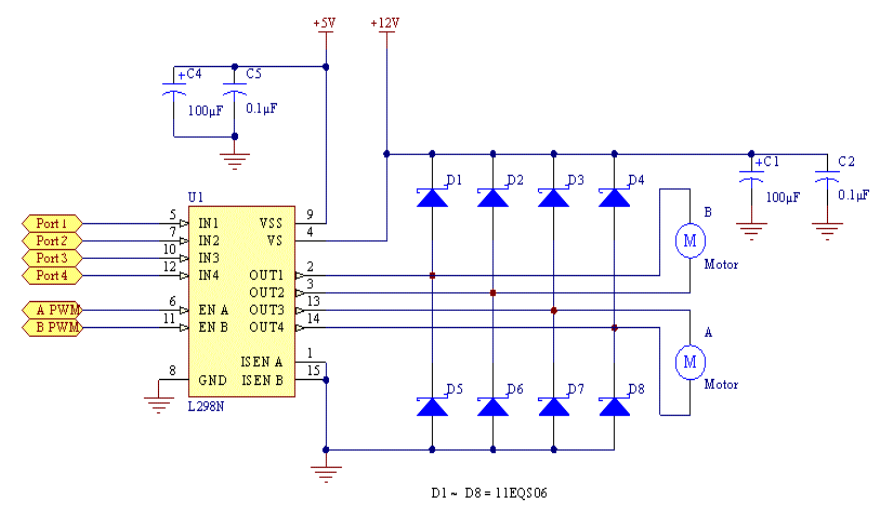

图 3-2 电机驱动电路

\section{4. 软件设计}

为了提高开发效率, 在编写程序时采用模块化的设计 
思想, 并采用当前应用最为广泛的 $\mathrm{C}$ 语言进行编写。单片 机最小系统主要完成检测边界、寻找对方、并将对方推出 界外的功能。

在本设计中, 所使用的控制器 Atmega128 属于 AVR 系 列, 我们为了在开发环境上更加方便, 我们采用的开发软 件为 iccv7ProLoader, 程序烧写软件为 AVR_fighter 这两个 软件使用起来非常方便, 并且稳定性能好, 其使用界面分 别如图 4-1 所示。

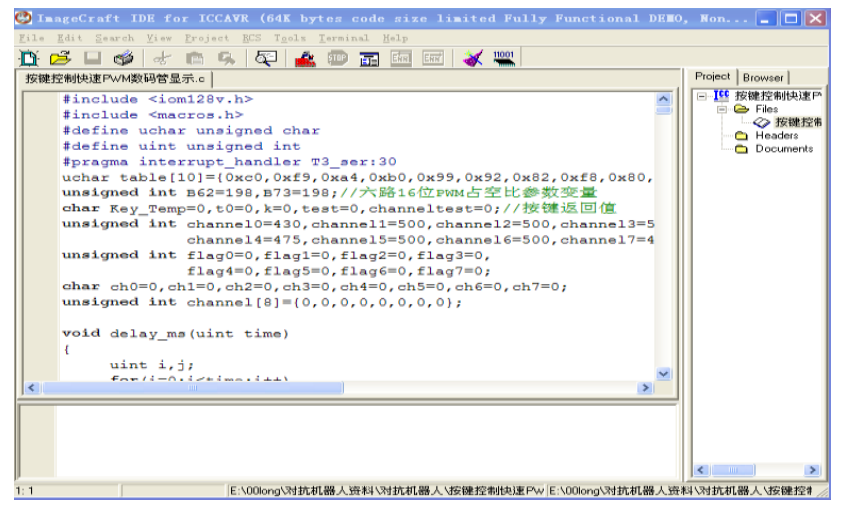

图 4-1 iccv7ProLoader 开发环境

\section{5. 系统整体调试}

在本系统中, 硬件和软件的设计我们均采用模块化设 计思想, 为我们后期的整机调试做好了准备。我们的调试 步骤如下:

第一步, 调试单片机最小系统。首先, 在电源正常工 作情况下调试数码管和按键, 使其能够正常工作, 为下面 的调试做好准备。例如, 数码管可以显示 PWM 的占空比 以及机器人当前状态等等。

第二步, 调试检测电路。我们借助示波器调试红外对 管, 确保每对红外对管能达到系统性能要求。

第三步, 调试电机驱动电路。电机驱动模块在没有接 单片机情况下, 利用多路直流稳压电源分别给 LN298 提供
电源和模拟信号, 然后判断电机驱动电路是否正常工作。 如果电机驱动电路不能正常工作, 依照原理图, 借助万用 表或示波器排查错误, 直到电机驱动电路可以正常工作。

第四步, 系统整机调试。在确保以上模块正常工作时, 将各个模块整体调试。

并且, 我们在硬件调试过程中, 也同时进行软件程序 的编写, 软件调试时先使用软件的仿真功能调试程序, 将 各模块程序调试成功。这样, 当硬件调试好后, 软件的仿 真也通过后, 我们就开始根据具体功能进行软、硬件联合 调试, 直至所有功能达到控制要求为止。

\section{6. 总结}

机器人可以检测边界、寻找对方、并将对方推出界外。 为了方便调试, 最小系统增设了键盘和显示电路, 提供良 好的人机交互接口, 方便调试, 这也使得整个系统非常人 性化。

但整个系统还存在一些不足, 例如: 为了使红外对管 检测距离更远, 减小了红外发射管的限流电阻, 最小甚至 达到 50 欧姆, 这样红外对管消耗的功率很大; 红外对管受 自然光影响比较大, 导致机器人误检测。这些都是以后需 要改进的地方 [4]。

\section{参考文献(References)}

[1] Selected singlechip technology, edited by He Limin, Beihang University press, 2006.

[2] $51 \mathrm{MCU}$ application development and practice, Chang Min, Wang Han, Fan Jiangbo et al., 2009.

[3] Principle and application of sensor, Wang Xuewen, edited by Zhang Zhiyong, Beihang University press, 2004.

[4] Atmega128 MCU application and development of the case, Zhang Qiusheng, Liu Lanxiang, ed., mechanical industry press, 2006. 